\title{
TECHNICAL CCD SYSTEMS FOR THE ESO VERY LARGE TELESCOPE
}

\author{
J. Ehrich \\ Jena-Optronik $\mathrm{GmbH}$
}

The Technical CCD Systems (TCCDS) consist of thermo-electrically cooled CCD detector heads together with the electronic hardware and software for the control of the CCD and readout of the data. Two different types of CCD heads equipped with a small and a large format CCD chip are used. The small format CCD systems will be used primarily for auto-guiding and field acquisition at the main foci of the VLT. The large format CCD systems are intended mainly for wavefront sensing applications. An overview of the primary modes of operation of the TCCDS and the corresponding fields and exposure conditions for these modes is given in Table 1. The term "Technical CCD Systems" is derived from the intended application in the VLT because due to the excellent CCD head parameters and the system performance a scientific application will be offered too.

\section{TABLE 1}

Primary modes of operation

\begin{tabular}{llll}
\hline \hline CCD Format & small & small & large \\
\hline Operational Mode & acquisition mode & guiding mode & wavefront sensing \\
Field Size & 1 arc min & $20 \times 20$ pixels & $\geq 12.5 \times 12.5 \mathrm{~mm}^{2}$ \\
Wavelength & $400-1000 \mathrm{~nm}$ & $600-700 \mathrm{~nm}$ & $00-600 \mathrm{~nm} ; 700-1000 \mathrm{~nm}$ \\
Integration Time & $0.5-2 \mathrm{sec}$ & $0.01-2 \mathrm{sec}$ & $\geq 30 \mathrm{sec}$ \\
Centroiding Accuracy & 0.2 pixel & 0.1 pixel \\
Limiting Magnitude & $>21$ & 16 (for $0.1 \mathrm{sec}$ int time) & 16 , distributed over 400 points \\
\hline
\end{tabular}

The CCD head (see Fig. 2) represents a compact mechanical design and is composed of the sealed box with a A/R coated glass window, the small or large CCD chip, the highly effective two stage Peltier cooler, two temperature sensors - one for the chip and the second for the box surface, hermetically sealed feed-through connectors, a liquid cooled heat exchanger, a external connector board with video preamplifier and cables to the array control electronic box. Furthermore the excellent performance of the CCD head is characterized by a minimum life time of 10 years, high positioning accuracy of the chip and high thermal stability to maintain the surface temperature of the head close (i.e., $\pm 1^{\circ} \mathrm{C}$ ) to ambient by using liquid cooling.

The CCD sensors are front illuminated frame transfer devices with a "2.5-phase" Virtual Phase image section. In comparison to conventional front illuminated CDDs the virtual phase 


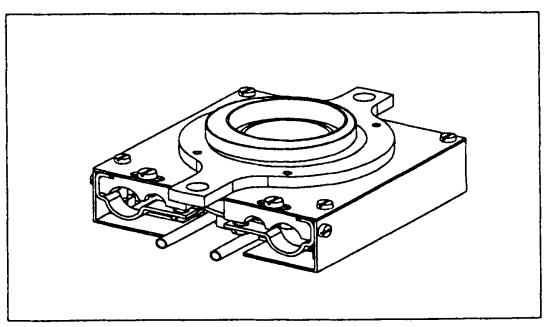

Fig. 1. Three-D view of the CCD head.

provides a wide spectral range (typically $200-1000 \mathrm{~nm}$ ) with enhanced blue sensitivity, very uniform photoelectric response and symmetric aperture response of a pixel in full sperctral range.

The TCCDS electronic hardware consists of two building blocks, the ACE box and an associated Local Control Unit (LCU). These units are joined by a $20 \mathrm{MHz}$ serial bi-directional transputer fiber-optic link.

At a distance of $2.5 \mathrm{~m}$ from the CCD head the array control electronics (ACE) is located in a compact box with an integrated liquid cooled heat exchanger. The ACE represents a modification of a design developed by ESO, especially the transputer based CCD controller. The ACE consists of three functional modules - sequencer, clock driver and video processor. The ACE provides universal control of different CCD detectors with varying clock diagrams, maximum 16 clock electrodes with 32 individually programmable voltage levels. The video processor with analogue pre-processing, 1, 2, 4, 8-gain switching, 12-bit A/D conversion and data acquisition with a 16-bit transputer provides a 12 bit dynamic range in any single integration or 14 bit with gain switching. In addition to these the ACE box contains a bus back plane, a power supply module, a module for auxiliary functions as temperature monitoring etc. and the fiber optic interface as one part of the data transfer link to the LCU. The other part , the link adapter board (LAB), represents a VME interface card located in the LCU and, like the fiber optic interface, converts electrical signals from/to the link interface board to optical signals for transmission to /from the ACE. The transputer serial link is interfaced to the VMEbus of the LCU via the link interface board (LIB). The LCU is a standard VMEbus processor which is connected to the main VLT computer system through a Local Area Network and is dedicated to the control of the TCCDS.

A comprehensive software package is provided. It consists of ACE embedded software and test software. The ACE embedded software is running on a transputer network. The CCD chip control will be done by the DSP 56001. On the LCU runs the control and test software under VxWorks. Command and control of the embedded software is realized from the LCU via a transputer link to ACE. This software package will be integrated in the VLT software developed by ESO running on different workstations.

The company Jena-Optronik GmbH, a subsidiary of Deutsche Aerospace and Jenoptik, has been chosen by the European Southern Observatory to develop and manufacture the Technical CCD Systems for the ESO Very Large Telescope. 